\title{
Review of the ultrafast time resolution photopolarimeters based on SPADs
}

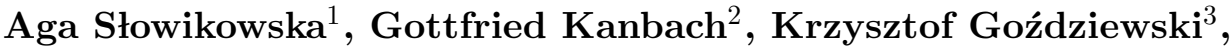 \\ Krzysztof Krzeszowski ${ }^{1}$ and Arne Rau ${ }^{2}$ \\ ${ }^{1}$ Kepler Institute of Astronomy, University of Zielona Góra, Zielona Góra, Poland \\ ${ }^{2}$ Max Planck Institute for Extraterrestrial Physics, Garching, Germany \\ ${ }^{3}$ Toruń Centre for Astronomy, Nicolaus Copernicus University, Toruń, Poland
}

\begin{abstract}
We review photopolarimeters that are based on the Single Photon Avalanche Diodes (SPADs) and were designed, built, developed, and extensively used for high time resolution studies of astrophysical sources. Examples of such detectors are OPTIMA, GASP, AquEYE, and IquEYE which can measure the time of arrival of single optical photons with an accuracy of down to 50 picoseconds. We describe the most exciting results obtained with the SPADs detectors starting from the best existing optical polarimetric measurements of the Crab pulsar, the discovery of the first optical magnetar and its quasi-periodic oscillations, as well as a verification of exoplanets around eclipsing cataclysmic variables. Additionally, we discuss possible applications of such detectors for asteroseismology.
\end{abstract}

Keywords. photometers, polarization, pulsars, magnetars, cataclysmic variables, exoplanets

\section{Single Photon Avalanche Diodes (SPAD) instruments}

OPTIMA - Optical Pulsar Timing Analyzer (Straubmeier 2001, Kanbach et al. 2003 and 2008, Stefanescu 2011) is a very high time resolution, single-photon sensitive optical photometer and polarimeter. It uses optical fibres to gather light from fixed apertures in the focal plane to SPADs, while the field surrounding the apertures is imaged using a standard CCD detector. Single photons are recorded with absolute UTC time-scale tagging accuracy of $\sim 5 \mathrm{~ns}$. The quantum efficiency of the SPADs reaches a maximum of $60 \%$ at $750 \mathrm{~nm}$ and lies above $20 \%$ in the range $450-950 \mathrm{~nm}$. The system was designed from scratch as a guest instrument, easily adapted to different telescopes. It can be reconfigured for photometric or polarimetric use within one observing run.

GASP - Galway Astronomical Stokes Polarimeter is a fast, full Stokes, astronomical imaging polarimeter. Its construction allows to measure all four elements of the Stokes vector (I, Q, U, V) simultaneously over a broad wavelength range (400-800 nm). Measuring linear and circular polarization with a time resolution of the order of microseconds makes GASP a unique astronomical instrument (Kyne et al. 2010).

IquEYE and AquEYE - ultra-high-speed photometers capable of tagging the arrival time of each photon with a resolution and accuracy of 50 picoseconds, for hours of continuous acquisition, and with a dynamic range of more than 6 orders of magnitude (Barbieri et al. 2009, 2012, Naletto et al. 2009).

All these detectors are portable and were used successfully at several observatories.

\section{Example results}

Crab pulsar and its nebula. The linear polarization of the Crab pulsar and its close environment was derived with a time resolution as short as $11 \mu \mathrm{s}$, which corresponds to a phase interval of $1 / 3000$ of the pulsar rotation $(33 \mathrm{~ms})$. High sampling allows 
to derive polarization details never achieved before (Słowikowska et al. 2009). These results were recently confirmed by Moran et al. (2013). On the other hand, AquEYE allowed the first sub-microsecond optical timing of the Crab (Germanà et al. 2012).

First optical magnetar. We observed extremely bright and rapid optical flaring in the Galactic transient SWIFT J195509.6+261406 (Stefanescu et al. 2008). Its optical light curves are phenomenologically similar to high-energy light curves of soft $\gamma$-ray repeaters and anomalous X-ray pulsars, which are thought to be neutron stars with extremely high magnetic fields - magnetars.

HU Aqr - a Jovian companion? The eclipsing binary HU Aqr is a polar (mWD + M4 V) with an orbital period of 125 minutes. This binary exhibits periodic variations of the observed-minus-calculated $(\mathrm{O}-\mathrm{C})$ curve. We performed a detailed study of archival and new OPTIMA observations, in terms of a new Light Travel Time (LTT) ephemeris model, formulated with respect to the Jacobi coordinates with the origin in the mass center of the binary. We found that the observations are best explained by one periodic signal, which can be interpreted by the presence of a $\sim 7$ Jupiter-mass planet, in a $\sim 10$ yr quasi-circular orbit (Goździewski et al. 2012, Słowikowska et al. 2013).

\section{Conclusions}

High time resolution instruments based on SPADs allow to time tag single optical photons with accuracy even down to 50 picoseconds. They have been successfully used to study rapidly changing sources such as optical pulsars, cataclysmic variables, including polars and intermediate polars (Nasiroglu et al. 2012), as well as very fast flares from optical magnetars. SPAD instruments can be successfully used also in case of pulsating stars to study different oscillation modes, especially at high frequencies. Such oscillations penetrate to different depths inside the stars and provide information about their otherwise unobservable interiors.

\section{Acknowledgements}

AS and KK acknowledge support from NCN, grant UMO-2011/03/D/ST9/00656.

\section{References}

Barbieri, C., Naletto, G., Capraro, I., et al. 2009, Proc. SPIE, 7355, 15

Barbieri, C., Naletto, G., Zampieri, L., et al. 2012, in: R. E. M. Griffin, R. J. Hanisch, \& R. Seaman (eds.), New Horizons in Time-Domain Astronomy, Proc. IAU Symposium No. 285 (Cambridge: Cambridge University Press), p. 280

Germanà, C., Zampieri, L., Barbieri, C., et al. 2012, A\& A, 548, A47

Goździewski, K., Nasiroglu, I., Słowikowska, A., et al. 2012, MNRAS, 425, 930

Kanbach, G., Kellner, S., Schrey, F. Z., et al. 2003, Proc. SPIE, 4841, 82

Kanbach, G., Stefanescu, A., Duscha, S., et al. 2008, ASSL, 351, 153

Kyne, G., Sheehan, B., Collins, P., Redfern, M., \& Shearer, A. 2010, EPJ Web of Conferences, 5, 5003

Moran, P., Shearer, A., Mignani, R. P., et al. 2013, MNRAS, 433, 2564

Naletto G., Barbieri, C., Occhipinti, T., et al. 2009, A\& $A, 508,531$

Nasiroglu, I., Słowikowska, A., Kanbach, G., \& Haberl, F. 2012, MNRAS, 420, 3350

Słowikowska, A., Kanbach, G., Kramer, M., \& Stefanescu, A. 2009, MNRAS, 397, 103

Słowikowska, A., Goździewski, K., Nasiroglu, I., et al. 2013, ASP-CS, 469, 363

Stefanescu, A. 2011, Ph.D. Thesis, Technische Universität München

Stefanescu, A., Kanbach, G., Słowikowska, A., et al. 2008, Nature, 455, 503

Straubmeier, C. 2001, Ph.D. Thesis, Technische Universität München 